\title{
Resveratrol inhibits glioma cell growth via targeting oncogenic microRNAs and multiple signaling pathways
}

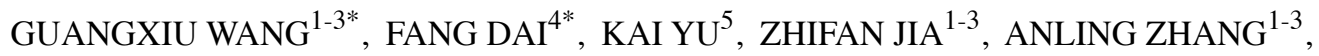 \\ QIANG HUANG ${ }^{2,3,5}$, CHUNSHENG KANG $^{1-3}$, HAO JIANG $^{6}$ and PEIYU PU ${ }^{1-3,5}$ \\ ${ }^{1}$ Laboratory of Neuro-Oncology, Tianjin Neurological Institute; ${ }^{2}$ Key Laboratory of Post-trauma Neuro-repair and \\ Regeneration in Central Nervous System, Ministry of Education; ${ }^{3}$ Tianjin Key Laboratory of Injuries, Variations and \\ Regeneration of Nervous System, ${ }^{4}$ State Key Laboratory of Applied Organic Chemistry, Lanzhou University, \\ Lanzhou, Gansu 730000; ${ }^{5}$ Department of Neurosurgery, Tianjin Medical University General Hospital, \\ Tianjin 300052, P.R. China; ${ }^{6}$ Department of Neurology, Henry Ford Hospital, Detroit, MI 48202, USA
}

Received November 12, 2014; Accepted December 29, 2014

DOI: $10.3892 /$ ijo.2015.2863

\begin{abstract}
Resveratrol (Res), a natural polyphenolic compound, has anticancer activity in a variety of cancers. In the present study, the antitumor effect and underlying molecular mechanism of Res on rat C6 glioma growth was studied. The results demonstrated that Res inhibited glioma cell proliferation, arrested cell cycle in $\mathrm{S}$ phase and induced apoptosis in vitro. Res also suppressed intracranial C6 tumor growth in vivo and prolonged survival in a fraction of the rats bearing intracranial gliomas. Res significantltly downregulated the specific miRs, including miR-21, miR-30a-5p and miR-19, which have been identified as oncomiRs in our previous studies, and altered the expression of their targeting and crucial genes for glioma formation and progression such as p53, PTEN, EGFR, STAT3, COX-2, NF- $\mathrm{kB}$ and PI3K/AKT/mTOR pathway. Therefore, the anti-glioma effect of Res, at least in part, is through the regulation of oncogenic miRNAs. The effect of Res on noncoding RNAs should be studied further. Res is a potential multi-targeting drug for the treatment of gliomas.
\end{abstract}

\section{Introduction}

Malignant gliomas are the most common and highly aggressive primary brain tumors. Even using multiple modalities of treatment, including maximal safe resection, radiotherapy, and chemotherapy, the prognosis of patients with malignant gliomas remains dismal $(1,2)$. Therefore, novel therapeutic strategies and drugs for treatment of malignant gliomas are urgently required.

Correspondence to: Professor Peiyu Pu, Department of Neurosurgery, Tianjin Medical University General Hospital, 154 An-Shan Road, Tianjin 300052, P.R. China

E-mail: pupeiyu33@hotmail.com

${ }^{*}$ Contributed equally

Key words: apoptosis, cell growth, glioma, microRNA, resveratrol
A large number of chemopreventive and chemotherapeutic agents have been discovered from natural products and provided promising approaches to treat and prevent cancer $(3,4)$. Res (3,5,4'-trihydroxy-trans-stilbene), a naturally polyphenolic compound, has been identified in the skin of red grapes, peanuts and various food products. It has a variety of important biological effects such as suppression of platelet aggregation, anti-inflammatory, anti-oxidant, and vasorelaxant activities (5-7). Res also exhibits antitumor properties by blocking the three stages of carcinogenesis, initiation, promotion and progression (8). Res has exerted anticancer effects against prostate, breast, leukemia and other epithelial cancer cells. There are some reports on the studies of the treatment of gliomas with Res (9-12) but the molecular mechanism of its antitumorigenic or chemopreventive activities is complex and not fully understood.

The objective of the present study was to investigate the anti-proliferative effect of Res on glioma cells using both in vitro and in vivo models. It is well known that small non-coding regulatory RNAs - microRNAs (miRs) are found to be dysregulated in almost all types of cancers and play important roles in cancer development and progression (13-17). Recent studies have demonstrated that miRs can be regulated by natural agents such as curcumin and Res resulting in suppression of tumor growth, drug resistance and metastasis (18-21). Our previous studies have confirmed that specific miRs, including miR-21, miR19 and miR-30a-5p are upregulated in GBMs with significant oncogenic activity and their major targeted genes involved in gliomagenesis (22-24). Antisense oligonucleotides of these miRs significantly suppress the glioma cell growth either in vitro or in vivo $(25,26)$. We examined the alteration of these specific miRs in GBM cells after treatment with Res, and also the expression of their targeted genes and the genes related to glioma formation and progression.

\section{Materials and methods}

Cell culture. Rat C6 glioma cells were obtained from the Institute of Biochemistry and Cell Biology, Chinese Academy 
of Sciences. Cells were cultured at $37^{\circ} \mathrm{C}$ with $5 \% \mathrm{CO}_{2}$ in Dulbecco's modified Eagle's medium (Invitrogen, USA) supplemented with $10 \%$ heat-inactivated fetal calf serum, $4 \mathrm{mM}$ glutamine, $50 \mathrm{U} / \mathrm{ml}$ penicillin and $50 \mu \mathrm{g} / \mathrm{ml}$ streptomycin (Invitrogen). Cells were subcultured every other day.

Cell proliferation examined by MTT (3-(4.5-dimethylthiazol2-yl)-2,5-diphenyltetrazolium bromide) assay. MTT were purchased from Sigma-Aldrich (St. Louis, MO, USA). Cells were implanted in 96 -well plates $(4,000$ cells/well) and allowed to grow overnight, then treated with 50,100 and $150 \mu \mathrm{M}$ of Res or $0.3 \%$ DMSO for $24,48,72$ and $96 \mathrm{~h}$, and exposed to $20 \mu \mathrm{l}$ MTT $(5 \mathrm{mg} / \mathrm{ml})$ at each time-point, then incubated for additional $4 \mathrm{~h}$. The resulting formazan crystals were solubilized with DMSO and optical density was measured in triplicate wells at the wavelength of $570 \mathrm{~nm}$. Results were expressed as percentage of control.

Cell cycle kinetic analysis. C6 glioma cells $\left(6 \times 10^{5}\right.$ cells/ well) were plated in $10-\mathrm{cm}$ dishes and incubated for $24 \mathrm{~h}$ DMSO $(0.3 \%)$ or different concentration of Res $(50,100$ and $150 \mu \mathrm{M}$ ) were added to the dishes and incubated for additional 48 h. Cells were trypsinized, washed with PBS, fixed with $70 \%$ ethanol for a minimum of $30 \mathrm{~min}$, and incubated with RNase A $(100 \mu \mathrm{g} / \mathrm{ml})$ for $30 \mathrm{~min}$ at $37^{\circ} \mathrm{C}$. Cells were then stained with propidium iodide in PBS $(50 \mu \mathrm{g} / \mathrm{ml})$ for $30 \mathrm{~min}$ at room temperature. Samples were analyzed by FACSCalibur flow cytometer and Modifit software (BectonDickinson, USA).

Detection of apoptotic cell death. The extent of apoptotic cell death was analyzed by using Annexin V-FITC Apoptosis Detection kit (BD Biosciences, USA). Cells $\left(6 \times 10^{5}\right.$ cells/dish) were cultured in $10-\mathrm{cm}$ dishes for $24 \mathrm{~h}$, treated with $0.3 \%$ DMSO and 50-150 $\mu \mathrm{M}$ of Res for $48 \mathrm{~h}$. Then both adherent and floating cells were harvested, double-labeled with Annexin V-PE and 7-amino-actinomycin, examined with FACSCalibur flow cytometer and analyzed by CellQuest software (Becton-Dickinson).

For xenograft tumor specimens, apoptosis was detected using TUNEL method with an in situ cell death kit (Roche, USA) according to the supplier's instructions. Stained cells were visualized using FluoView Confocal Laser Scanning Microscopes-FV1000 (Olympus, Tokyo, Japan) and analyzed using IPP5.1 (Olympus).

$q R T-P C R$. Total RNA from cells treated with $0.3 \%$ DMSO and different concentration of Res $(50,100$ and $150 \mu \mathrm{M})$ for $48 \mathrm{~h}$ were extracted using TRIzol reagent (Invitrogen). For analysis of miR expression, real-time RT-PCR analyses were carried out using Hairpin-it ${ }^{\mathrm{TM}}$ miRNAs qPCR Quantitation kit (Shanghai GenePharma Co., Ltd., China) according to the manufacturer's instructions. Amplification reaction was performed with MJ-real-time PCR (Bio-Rad, Hercules, CA, USA). Relative expression was calculated using the $\Delta \Delta \mathrm{CT}$ method and normalized to the expression of U6 endogenous control RNA and analyzed initially using Opticon Monitor Analysis V2.02 software (MJ Research, Waltham, MA, USA). All qRT-PCRs were performed in triplicate, and the data are presented as means \pm standard errors of the means (SEM).

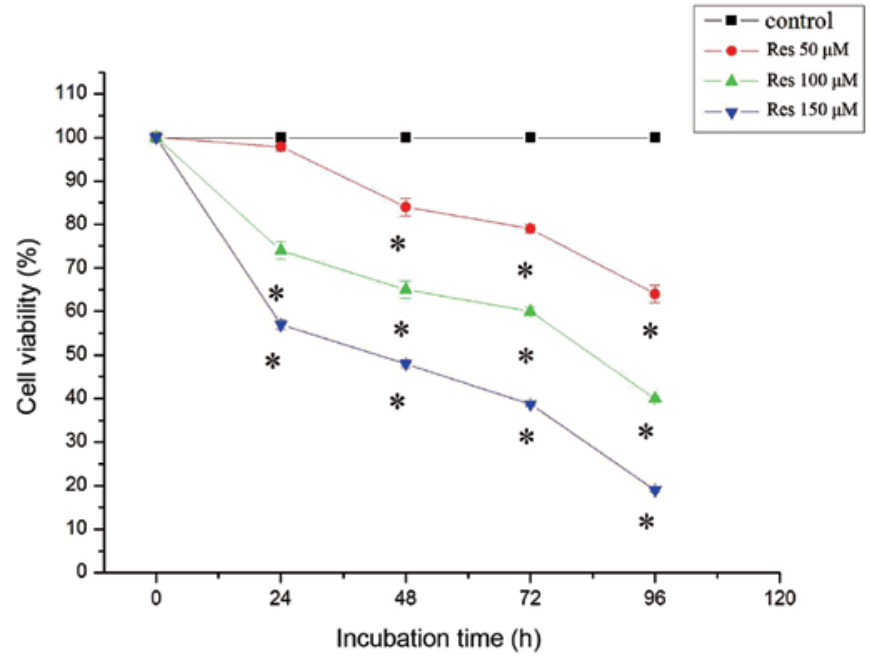

Figure 1. The effect of Res on survival rate of C6 cells detected by MTT assay. The dose- and time-dependent inhibition of cell growth was observed in C6 cells treated with different concentrations of resveratrol (50, 100 and $150 \mu \mathrm{M}) .{ }^{*} \mathrm{p}<0.01$.

Western blot analysis. Total proteins were extracted from cells treated with $0.3 \%$ DMSO or 50,100 and $150 \mu \mathrm{M}$ of Res for $48 \mathrm{~h}$. The protein concentration was determined by Lowry method. Protein $(40 \mu \mathrm{g})$ was subjected to SDS-PAGE. Separated proteins were transferred to PVDF membranes (Millipore, USA), followed by incubation for $1 \mathrm{~h}$ in blocking buffer. The membranes were then incubated separately with primary antibodies against EGFR, PI3K, p-AKT, mTOR, PTEN, VEGF, PCNA, Cyclin A, cyclin E, Bcl-2, Caspase-3, GFAP, NF- $\kappa B$, Stat3, COX-2 (1:500 dilution, Santa Cruz Biotech, USA), followed by incubation with HRP-conjugated secondary antibodies (1:500 dilution, Zymed, USA). The specific proteins were detected using a SuperSignal protein detection kit (Pierce, USA). After washing with stripping buffer, the membranes were reprobed with an antibody against $\beta$-actin (1:500 dilution, Santa Cruz) following the procedures described above. The relative quantification was expressed as the density of specific proteins divided by the density of $\beta$-actin.

Immunohistochemical staining. After Res treatment, cells were immunostained for MMP-9 or NF- $\kappa$ B by ABC-peroxidase method. Briefly, cells grown on the coverslips for $24 \mathrm{~h}$ were treated with DMSO $(0.3 \%)$ or Res $(100 \mu \mathrm{M})$ for $48 \mathrm{~h}$, then fixed with $4 \%$ paraformaldehyde, treated with $3 \%$ hydrogen peroxide and incubated with appropriate antibodies (1:1,000 dilution, Santa Cruz) overnight at $4^{\circ} \mathrm{C}$. After incubation, biotinylated secondary antibody (1:1,000 dilution, Zymed, USA) was added and incubated for $1 \mathrm{~h}$ at room temperature, followed by incubation with $\mathrm{ABC}$-peroxidase for additional $1 \mathrm{~h}$. Then washing with Tris-buffer, the cells were incubated with DAB (3,3' diaminbenzidine, $30 \mathrm{mg}$ dissolved in $100 \mathrm{ml}$ Tris-buffer containing $0.03 \%$ hydrogen peroxide) for $5 \mathrm{~min}$, rinsed in water and counterstained with hematoxylin.

Tumor growth in vivo. Rat models bearing intracranial C6 glioma were established as described previously (25). All 

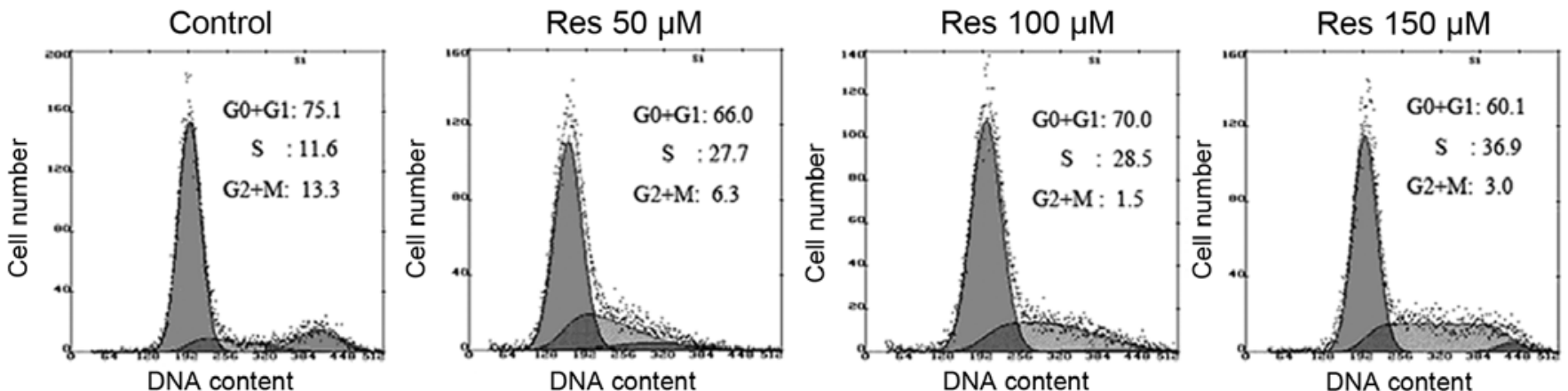

Figure 2. The effect of Res on cell cycle kinetics of C6 glioma cells examined by flow cytometry and showing the cells arresting in S phase.
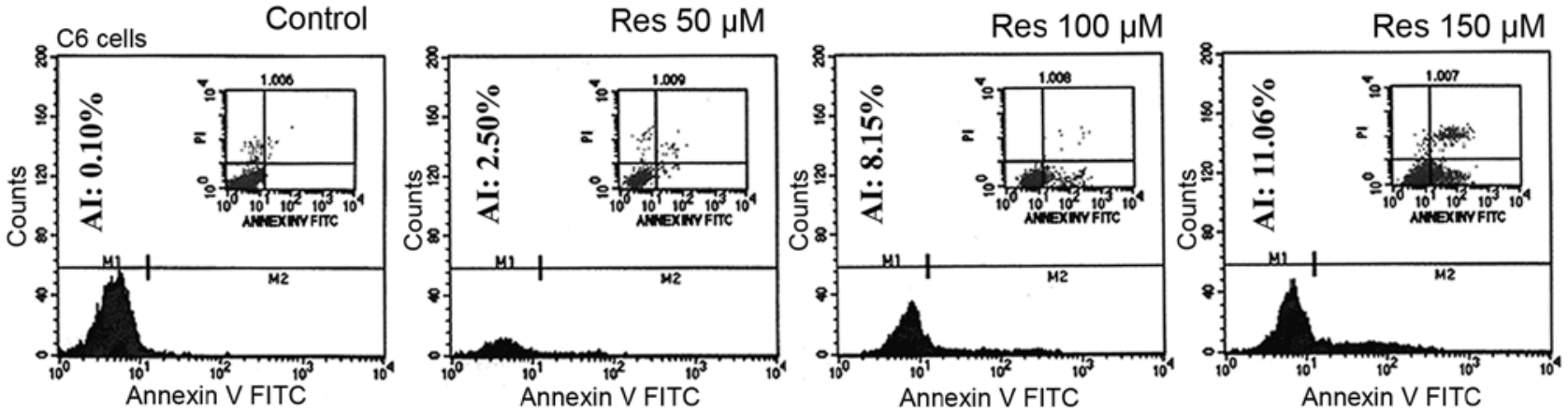

Figure 3. Induction of apoptosis in C6 cells treated with Res detected by flow cytometry.

animal experiments were approved by the Animal Care and Use Committee of Tianjin Medical University (Tianjin, China). Rats were randomly divided into two groups: control (10 rats) and treated (8 rats). In treated group, rats were given Res $8 \mathrm{mg} / \mathrm{kg} /$ day by oral administration until the end of observation period. The general behavior and survival of the rats were observed. The tumor growth in each group of rats were monitored at regular intervals using high-resolution MRI in order to compare the tumor volume at different periods in each individual animal. Whenever the rats in the control or Res treated group died naturally or were sacrificed at various time-points, their brains were removed and prepared as paraffin-embedded sections for histopathological examination and immunohistochemical staining. The expression of EGFR, GFAP, PCNA, MMP-9, NF- $\mathrm{B}, \mathrm{COX}-2$ and VEGF was detected by immunohistochemistry. The primary antibodies (1:1,000 dilution, Santa Cruz) used and the procedures were the same as described above.

Statistical analysis. The results are expressed as mean \pm SD. A Student's t-test was used to compare individual data with control value. A probability of $\mathrm{p}<0.05$ was considered to be significantly different from control data.

\section{Results}

Res inhibits glioma cell growth. The survival rate of C6 glioma cells treated with Res was measured by MTT assay. As shown in Fig. 1, the suppressive effect of Res on the proliferation of $\mathrm{C} 6$ cells was dose- and time-dependent.
After treatment with $150 \mu \mathrm{M}$ Res for 24 and $96 \mathrm{~h}$, the survival rate of C6 glioma cells declined to $58 \pm 1.26$ and $19 \pm 1.52 \%$, respectively, as compared to the control.

Res arrests the cell cycle. The effect of Res on cell cycle kinetics was analyzed by flow cytometry. The data obtained from cells treated with $0.3 \%$ DMSO and 50, 100 and $150 \mu \mathrm{M}$ of Res for $48 \mathrm{~h}$ are summarized in Fig. 2. Two-to-three fold increase of $\mathrm{S}$ phase fraction was demonstrated in C6 cells treated with different concentration of Res as compared to the control. These results suggested that Res was able to delay the cell cycle progression and inhibited the cell proliferation by arresting the cells cycle at $\mathrm{S}$ phase.

Res induces apoptotic cell death. The effect of Res on the apoptosis of glioma cells was determined by Annexin V-PE immunofluorescence staining. Apoptosis in glioma cells treated with Res was induced and the population of apoptotic cells was significantly increased in a dose-dependent manner. The percentage of apoptotic cells increased from 2.50 to $11.06 \%$ in C6 cells treated with $50-150 \mu \mathrm{M}$ of Res (Fig. 3).

Res downregulates the expression of miR-21, miR-30a-5p, miR-19 and their targeted or related components. qRT-PCR and the comparative $\mathrm{Ct}(\Delta \Delta \mathrm{Ct})$ method were used to determine the change of miR-21, miR-19 and miR30a-5p expression in C6 glioma cells before and after treatment with different concentration of Res. It was found that the expression of all these miRs was significantly downregulated in Res treated cells and also in a dose-dependent manner (Fig. 4). 

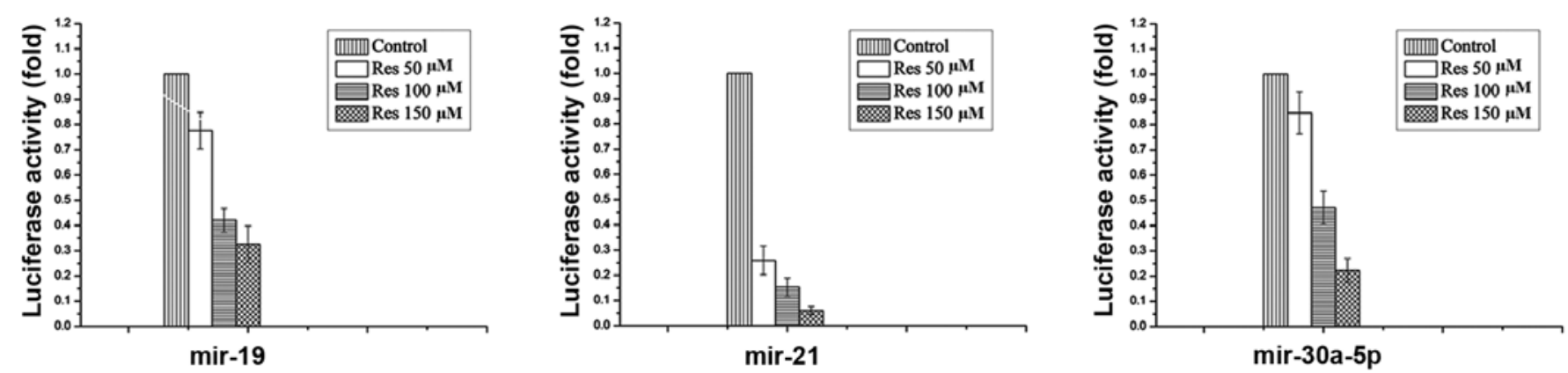

Figure 4. The expression of miR-21, miR-19 and miR30a-5p in control C6 glioma cells and cells treated with Res detected by qRT-PCR.

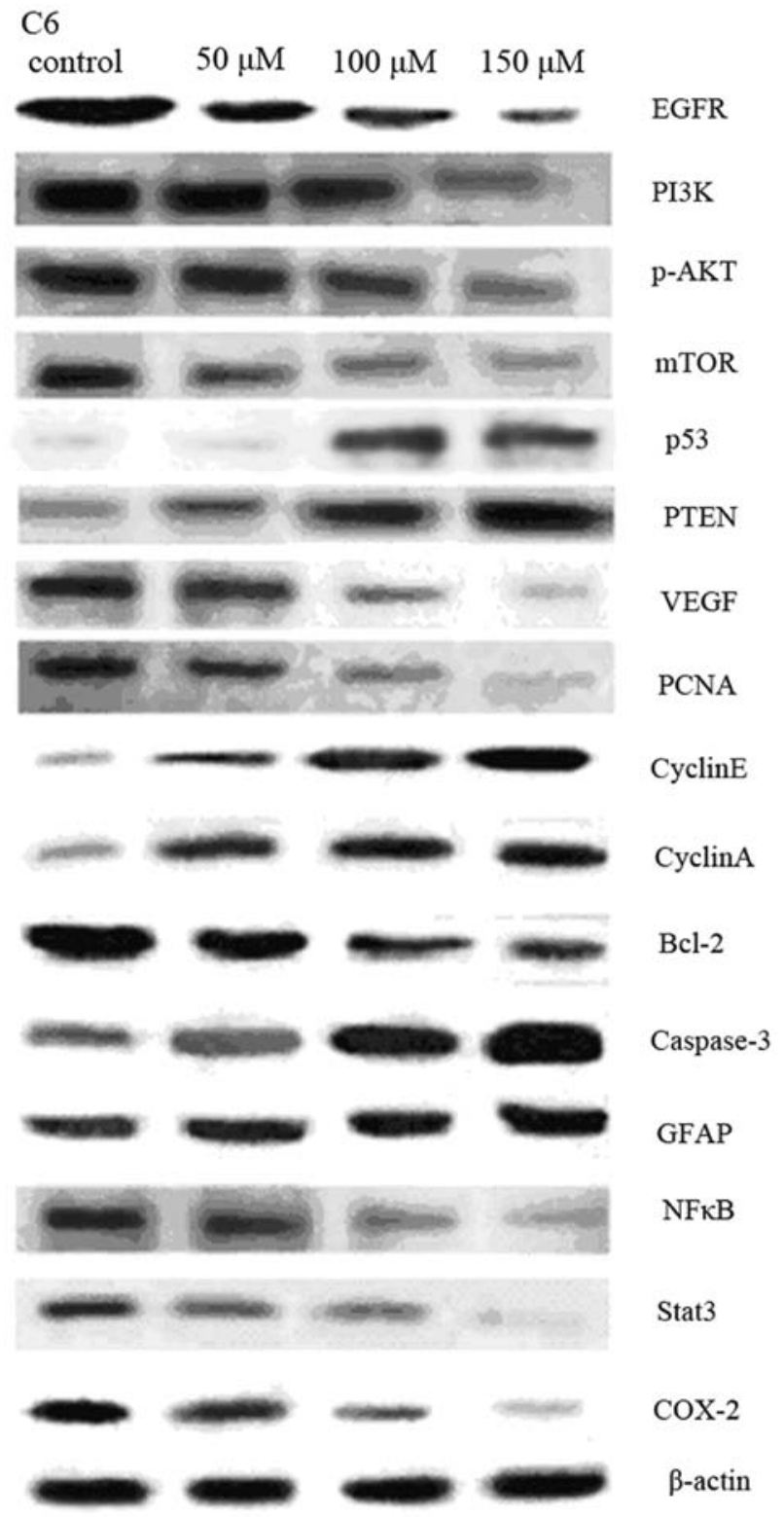

Figure 5. The expression of multiple genes in parental C6 cells and C6 cells treated with Res detected by western blotting.

Western blot analysis demonstrated that the expression of EGFR, PI3K, p-AKT, mTOR, VEGF, PCNA, Bcl-2, NF- $\mathrm{B}$, STAT3, COX-2 was downregulated, whereas the expression of p53, PTEN, Cyclin A, cyclin E, GFAP and Caspase-3 was upregulated in glioma cells treated with 50,100 and $150 \mu \mathrm{M}$ of Res (Fig. 5). In addition, MMP-9 and NF- $\kappa \mathrm{B}$ expression were decreased as shown by immunohistochemical staining (Fig. 6).

Antitumor effect of Res in rat C6 glioma xenograft. In vivo experiment showed that the general condition of rats was deteriorated at one week after injection of parental C6 glioma cells. The mean survival time of rats in control group was $15.80 \pm 0.93$ days (10 rats died on days $12,13,13,14,15,16$, 17, 18, 19 and 21, respectively). Eight rats receiving Res treatment had a longer survival than the control rats, with a mean survival of $29.75 \pm 9.27$ days ( 8 rats died on days $13,14,15,17$, $21,25,43$ and 90, respectively). There was a statistical difference between the mean survival time of the treatment group and the control group $(\mathrm{p}<0.05)$ (Fig. 7).

Two out of eight rats treated with Res had more favorable response. Their general conditions returned to normal at $\sim 4$ weeks after treatment. In one of them the primary tumor focus disappeared on day 73 and in another longer survival rat the tumor disappeared on day 35 as shown by MRI (the rats were sacrificed on days 90 and 43, respectively). Histopathological examination of their brains showed no macroscopic gliomas but only a tiny amounts of tumor cell residue in the original tumor region (Fig. 8). These results indicated that Res could inhibit tumor growth and prolong the survival in a fraction of the rats bearing intracranial $\mathrm{C} 6$ gliomas.

Besides, there were no apoptotic cells found in the tumor specimens of control rats. However, increased number of apoptotic cells could be observed in the tumors of Res-treated rats as detected by TUNEL method (Fig. 9).

The immunohistochemical staining of rat brain tumors treated with Res also demonstrated that the expression of EGFR, MMP-9 NF- $\kappa$ B, PCNA, COX-2 and VEGF was decreased, whereas the expression of GFAP was increased as compared with those in control rats (Fig. 9). These results suggested that multiple signaling molecules might be modulated by Res.

\section{Discussion}

The present study investigated anti-glioma effect of Res and its underlying molecular mechanism. Our results demonstrate that Res exerts a significant inhibitory effect on C6 glioma cell growth in a dose- and time-dependent manner. This anti- 


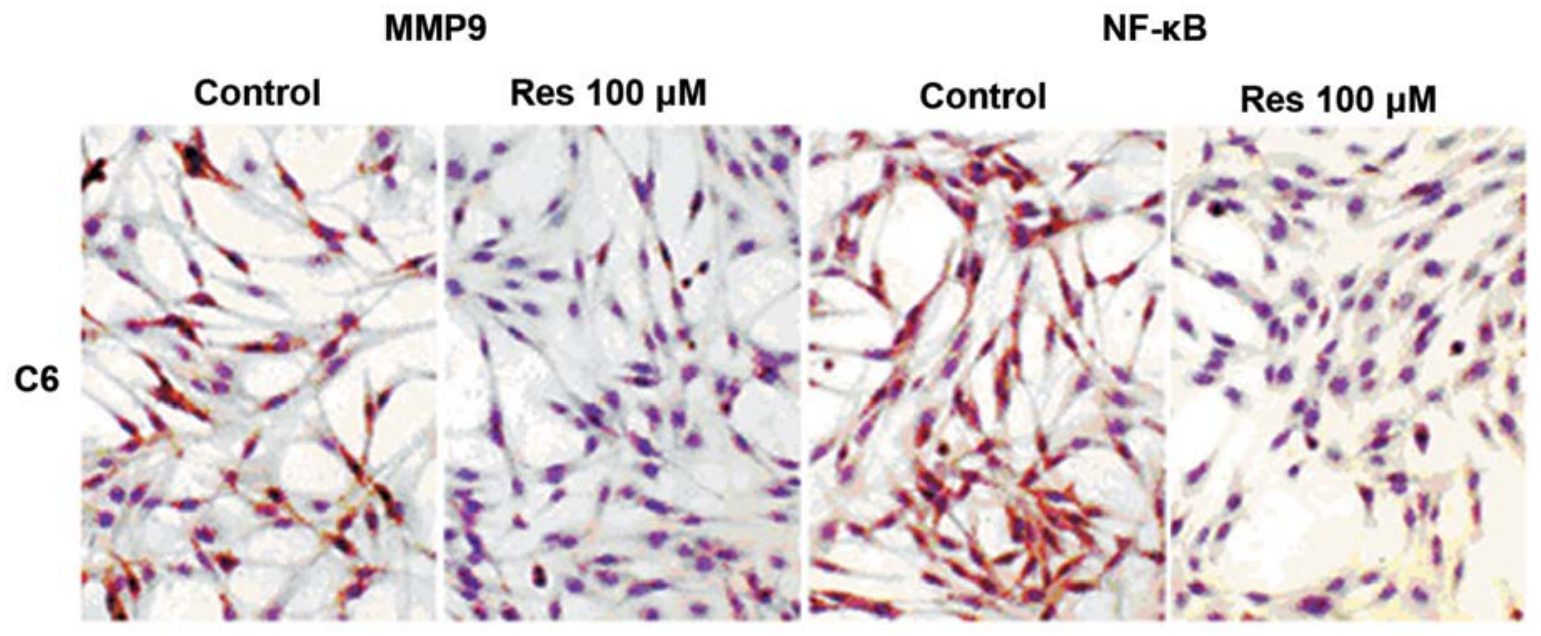

Figure 6. Immunohistochemical staining of MMP-9 and NF- $\mathrm{B}$ in parental C6 cells and cells treated with Res.

Survival of data: survival proportions

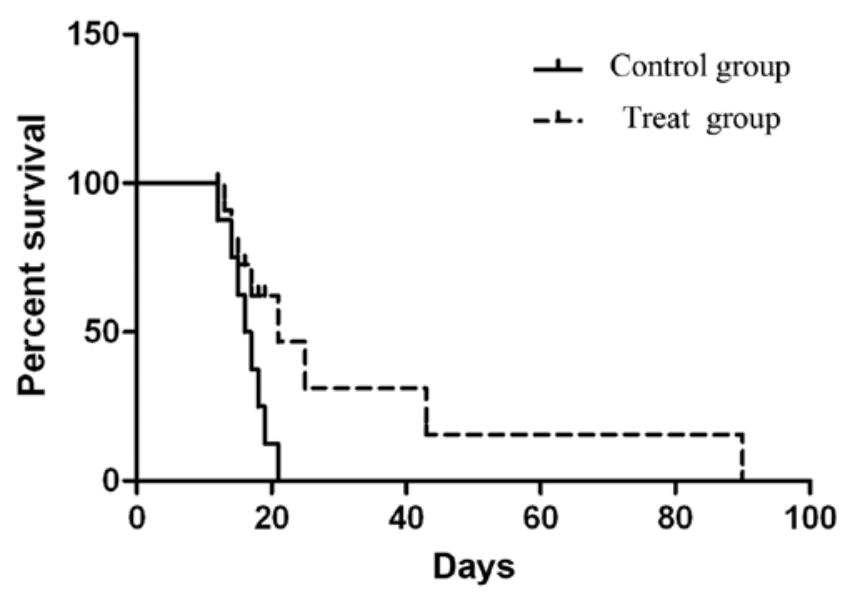

Figure 7. Kaplan-Meier survival rate analysis of rats bearing intracranial C6 gliomas untreated and treated with Res.

proliferative effect appears to result from the cell cycle arrest at S-phase and induction of apoptotic cell death. These results are in accordance with the findings from previously reported studies (27-29). Limited information is available on the antiglioma effect of Res in vivo. In this study, we found that the survival of a fraction of rats bearing intracranial gliomas treated with Res is prolonged. Among them, two rats had more favorable response to Res. These results indicate that Res is able to inhibit glioma growth both in vitro and in vivo, which is similar to the study of the suppressive effect of Res on the tumor growth of RT-2 glioma in vitro and in vivo (11). In addition, Gao et al reported that Res has strong anti-proliferative and pro-apoptotic effects against 32Dp210 leukemic cells in vitro, but Res at doses of $8 \mathrm{mg} / \mathrm{kg} / \mathrm{day}$ and $40 \mathrm{mg} /$ $\mathrm{kg} /$ day (oral administration for 5 days/week throughout the experiment) is ineffective in slowing down the progression of leukemia, as the doses escalated to $80 \mathrm{mg} / \mathrm{kg} / \mathrm{day}$, Res can prevent a small fraction of mice from leukemia-induced death (7). Based on our previous experiments on the rat C6 glioma models, the intracranial C6 gliomas shown by MRI could not regress spontaneously without appropriate treatment. It is conceivable to suggest that Res could inhibit tumor growth in a fraction of rat C6 gliomas. However, the dose of Res we used for the treatment of xenografts in vivo is low, whether the escalated doses of Res will enhance the suppression of glioma growth should be further studied.

Res has been shown to have antioxidant, anti-inflammatory, cell cycle arrest and cell death-inducing properties. Therefore, it is predictable that multiple targets and complex molecular events may be involved in Res-mediated inhibiton of glioma cell growth.

Alterations of a number of miRNAs play an oncogenic or tumor suppressor role in glioma formation and progression. Our previous studies have shown that miR-21, miR-19 and miR-30a-5p are overexpressed in gliomas and glioma cell lines, and involved in gliomagenesis as oncogenic miRs through their targeting genes. Antisense oligonucleotides of these miRs significantly suppress the glioma cell growth either in vitro or in vivo. The first study of Res affecting endogenous miRNAs was conducted in a human colon cancer cell line, Res was identified to decrease the levels of oncogenic miRNAs, including miR-17, -21, -23a/b, -146a and -103-1/2, and increase the level of tumor suppressor miR-663 (30). We also found that Res is able to inhibit the expression of oncogenic miR-21, miR-19 and miR-30a-5p in glioma cells.

MiR-21 overexpression in gliomas and glioma cell lines is a general consensus. It has been identified as a predominant microRNA altered in gliomas and play a crucial role in a myriad of biological processes. To our current knowledge, miR-21 targets sets of genes associated with tumor suppressive and pro-apoptotic function, such as PTEN, p53, TGF- $\beta$, PDCD4, TPM1 and Reck (31-34). MiR-21 expression is also correlated with downstream factors such as Bcl-2 and MMP2, which may represent indirect targets affected by the expression levels of miR-21, since there is no evidence of direct interaction between miR-21 and 3'UTR of their mRNA.

The expression and function of miR-19 and miR-30a-5p in gliomas have not been reported before. However, PTEN, the target of miR-19 we identified in glioma cells (23), and also reported as the target of $\mathrm{miR} 19 \mathrm{a} / \mathrm{b}$ in gastric cancer 

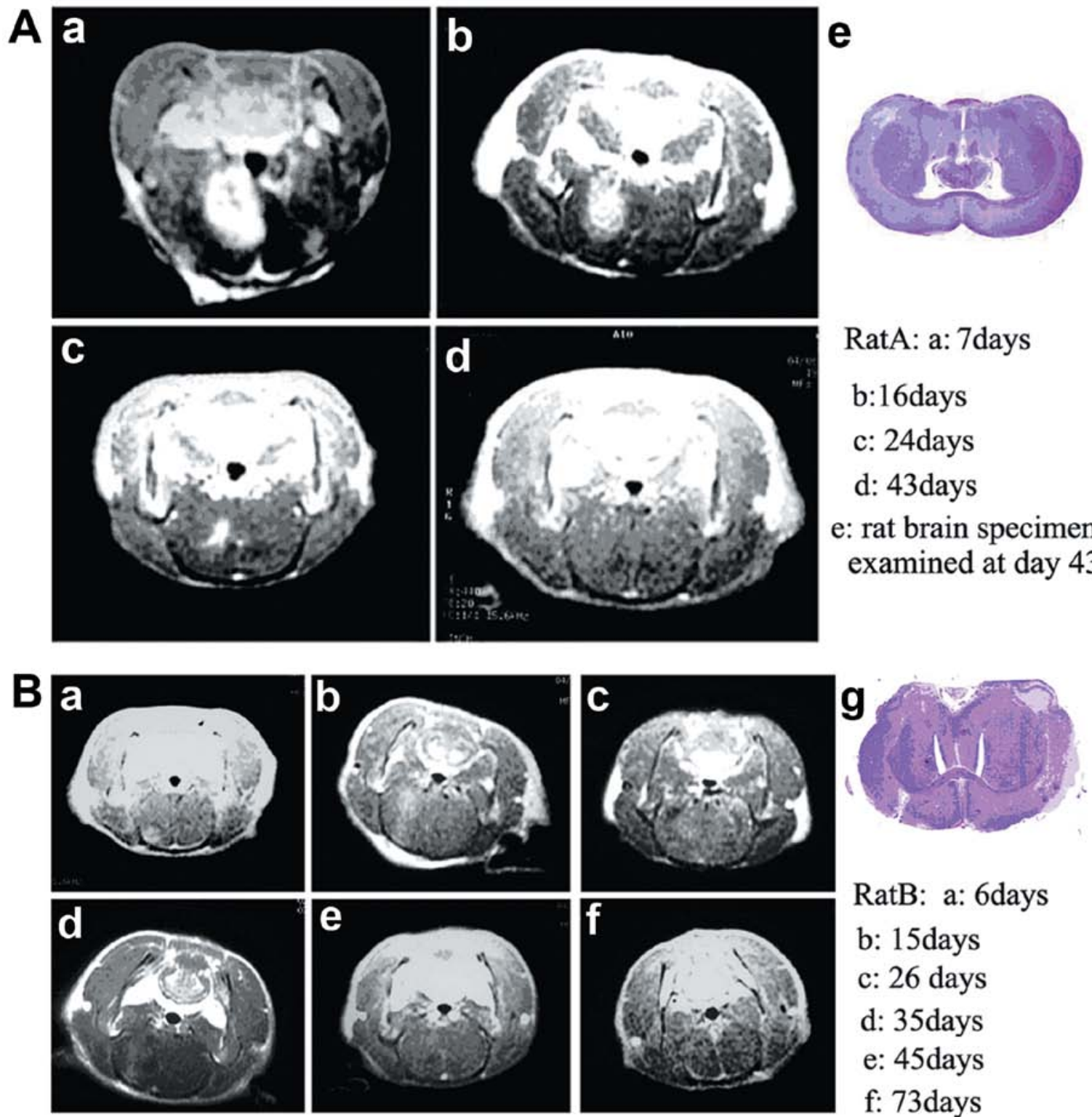

\section{RatB: a: 6days}

b: 15 days

c: 26 days

d: 35 days

e: 45 days

f: 73days

g: rat brain specimen

examined at day 90

Figure 8. The MR images of intracranial C6 gliomas in the rats treated with Res ( $8 \mathrm{mg} / \mathrm{kg} / \mathrm{day})$ survived for 43 and 90 days, respectively.

(35). Besides, miR-30 family members have been found to regulate cell apoptosis through the p53-Drp1 (dynamin-related protein 1) pathway.

PTEN is the common target of miR-21 and miR-19 and the critical tumor suppressor in GBM (24). As shown in Fig. 6, Res suppresses miR-21 and miR-19 may induce PTEN activation and results in repression of the major oncogenic PI3K/AKT/ mTOR signaling pathway in glioma cells, leading towards inhibition of tumor cell proliferation, invasion and induction of apoptosis. p53 is also the major target of miR-21, and tumor suppressor p53 deletion and mutation is also an important molecular event in gliomas. As shown in the present study, we demonstrated that $\mathrm{p} 53$ expression is significantly upregulated after treatment with Res. So that Res suppresses glioma cell growth, at least in part, through downregulation miR-21 and miR-19 and consequently, upregulating PTEN and p53.
EGFR overexpression is one of the most important genetic aberrations in malignant gliomas. A number of studies have demonstrated that EGFR is overexpressed in $60-90 \%$ of glioblastomas (36), as well as in C6 glioma cells (25). Hyperactivated EGFR signaling promotes cell growth and inhibits apoptosis via its major downstream and crosstalk signaling pathways, such as PI3K/AKT/mTOR and RAS/MAPK, which can regulate cell survival, proliferation, invasion and angiogenesis (37-39). Our previous studies have demonstrated that antisense and dominantnegative EGFR cDNA or siRNA targeting EGFR effectively inhibit glioma cell growth $(25,40)$. It has also been found that there is a significant correlation between EGFR and miR-21 levels in glioma cells. Antisense miR-21 markedly reduced EGFR expression in our previous study (24). Thus, the overexpression of EGFR is decreased in C6 glioma cells after treatment with Res, may be partially through the inhibition of miR-21. 


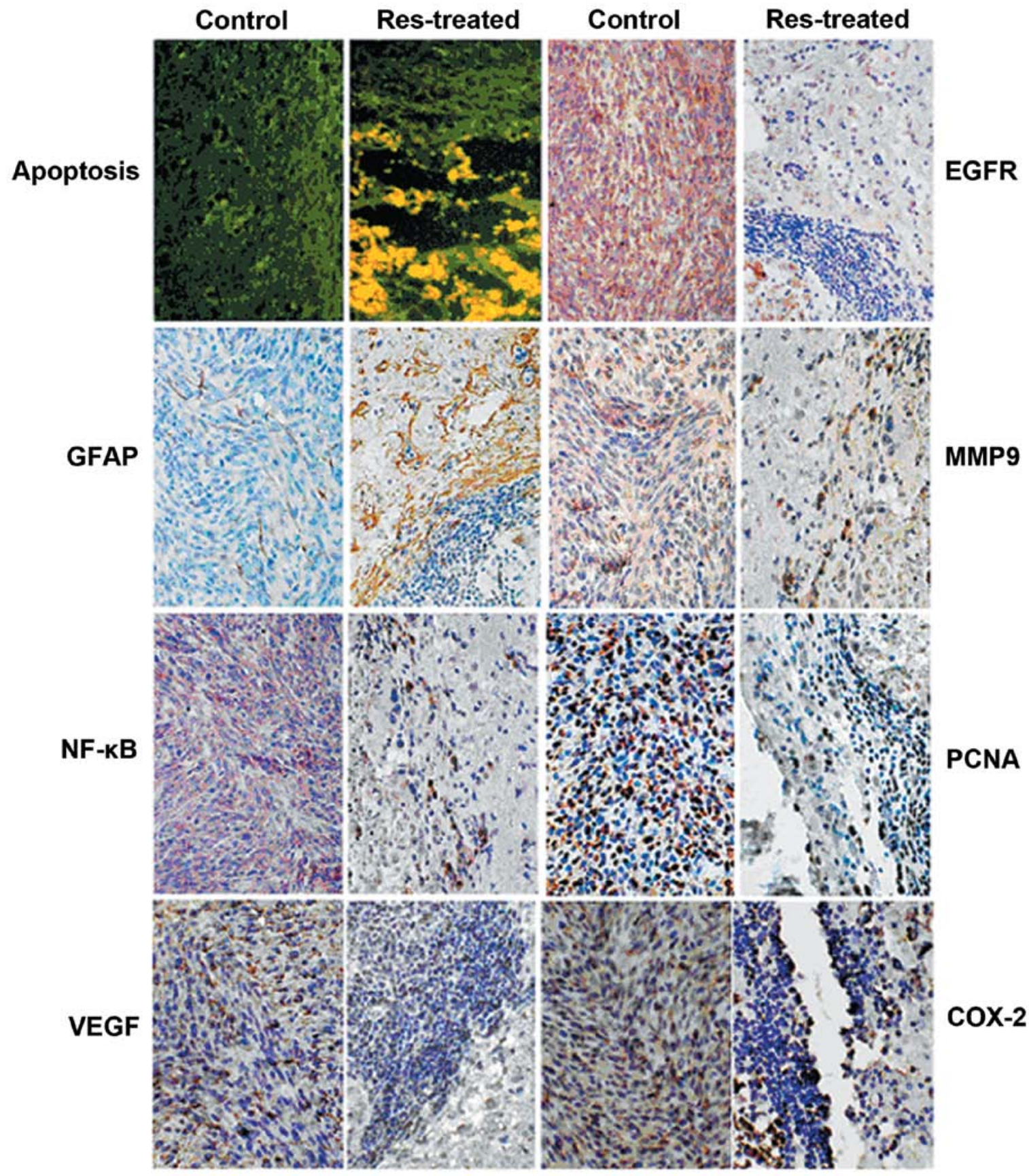

Figure 9. The expression of EGFR, PCNA, NF-кB, GFAP, COX-2, VEGF and MMP9 detected with immunohistochemical staining and the apoptotic cells determined by TUNEL method in rat intracranial C6 glioma untreated and treated with Res.

STAT3 overactivation is associated with a variety of cancers, including gliomas, and is identified as a master regulator of biological processes leading to the cancer formation (41-43). The present study demonstrates that Res significantly represses the expression of STAT3. This is one of the major reversions of aberrant molecular events in glioma cells following treatment with Res. It has been reported that Res suppresses constitutively active STAT3 in all the NK cell lines by inhibiting JAK2 phosphorylation but not other upstream mediators of STAT3 activation.

We have found previously that STAT3 binds to the miR-21 promoter by CHIP analysis and the STAT3 inhibitor significantly reduced miR-21 expression (44). Thus, STAT3 activation suppressed by Res may contribute to the reduced expression of miR-21.

The anti-inflammatory property of Res results from its inhibitory effect on the synthesis of pro-inflammatory mediator COX-2, and downregulation of COX-2 is also mediated by the inhibitory effects of Res on the important nuclear transcription factor NF- $\mathrm{NB}$. COX-2 overexpression has been documented in malignant gliomas and strongly correlated with the poor prognosis of glioblastomas (45). COX-2 catalyzes the conversion of free arachidonic acid to prostaglandins. Prostaglandins can stimulate tumor cell proliferation, promote angiogenesis, and suppress apoptosis. Res significantly inhibits the expression of COX2 and NF- $\mathrm{NB}$ either in vitro or in vivo. The suppres- 
sive effect of Res on COX-2 is also suggested as one of the molecular mechanisms responsible for its antiglioma activity. Additionally, NF- $\mathrm{KB}$ is constitutively activated in a number of cancers, including gliomas, Res can downregulate the expres-

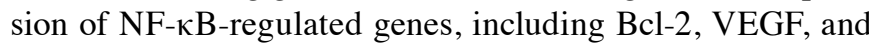
MMP-9, through which it may also affect apoptosis and invasion of glioma cells. Banerjee et al showed that Res inhibits the activation of NF- $\mathrm{KB}$ in breast cancer cells and dietary administration of Res in rats bearing mammary tumors inhibits tumor growth and decreases the expression of NF- $\mathrm{kB}$, COX-2 and MMP-9 in tumor tissues (46). It is consistent with our findings that the expression of NF- $\mathrm{\kappa B}, \mathrm{COX}-2$, MMP-9 and VEGF is decreased in glioma cells after Res treatment.

Recently, it has been reported that Res can downregulate lncRNA (long non-coding RNA) MALAT-1 that results in the inhibition of Wnt/ $/$-catenin signaling pathway and suppression of human colorectal cancer cell invasion and metastasis (47), whereas $\mathrm{Wnt} / \beta$-catenin signaling pathway is also an important oncogenic pathway to promote glioma cell growth as we reported before (48). Thus the effect of Res on non-coding RNAs should be studied in more detail.

In addition, the preclinical studies have shown that Res enhances the antiglioma effect of temozolomide (TMZ) via ROS-dependent AMPK-TSC-mTOR signaling pathway and inhibiting autophagy, or Res reverses TMZ resistance via downregulation of MGMT. It has also been reported that Res sensitizes GBM tumor initiating cells to radiotherapy by inhibition of STAT3 axis (49-52).

Taken together, the results from the present study demonstrate that Res could inhibit cellular proliferation and potentiate apoptosis in rat C6 glioma cells both in vitro and in vivo. Based on the interfering with the expression of several oncogenic miRs and a panel of genes of signaling pathways involved in the processes of gliomagenesis and possibly sensitizing chemo- and radiotherapy for gliomas after treatment with Res, it is conceivable that Res could be classified as a multi-targeting agent.

Since Res is able to cross the blood-brain barrier and incorporate into brain tissue, and its toxicity is minimal even high doses used long-term in rats and mice $(3,53)$. Therefore, clinical trials in human gliomas are urgently required. Currently, analogs of Res with improved bioviability are being developed as potential anticancer agents, and clinical trials of Res against some other cancers are being conducted $(54,55)$. It is expected that Res will provide at least an adjuvant for currently available combined therapy against malignant gliomas.

\section{Acknowledgements}

This study was supported by the Natural Science Foundation of China (no. 81101915).

\section{References}

1. Kevin P, Becker, Yu J, et al: Status quo - standard-of-care medical and radiation therapy for glioblastoma. Cancer J 18: 12-19, 2012.

2. Yang P, Wang Y, Peng X, et al: Management and survival rates in patients with glioma in China (2004-2010): a retrospective study from a single-institution. J Neurooncol 2: 259-266, 2013.

3. Aggarwal BB, Bhardwaj A, Aggarwal RS, et al: Role of resveratrol in prevention and therapy of cancer: preclinical and clinical studies. Anticancer Res 24: 2783-2840, 2004.
4. Cao Y, Wang F, Liu HY, et al: Resveratrol induces apoptosis and differentiation in acute promyelocytic leukemia (NB4) cells. J Asian Nat Prod Res 7: 633-641, 2005.

5. De la Lastra CA and Villegas I: Resveratrol as an anti-inflammatory and anti-aging agent: mechanisms and clinical implications. Mol Nutr Food Res 49: 405-430, 2005.

6. Castello L and Tessitore L: Resveratrol inhibits cell cycle progression in U937 cells. Oncol Rep 13: 133-137, 2005.

7. Gao X, Xu YX, Divine G, et al: Disparate in vitro and in vivo antileukemic effects of resveratrol, a natural polyphenolic compound found in grapes. J Nutr 132: 2076-2081, 2002.

8. Jang M, Cai L, Udeani GO, et al: Cancer chemopreventive activity of resveratrol, a natural product derived from grapes. Science 275: 218-220, 1997.

9. Gagliano N, Moscheni C, Torri C, et al: Effect of resveratrol on matrix metalloproteinase-2 (MMP-2) and Secreted Protein Acidic and Rich in Cysteine (SPARC) on human cultured glioblastoma cells. Biomed Pharmacother 59: 359-364, 2005.

10. Jiang H, Zhang L, Kuo J, et al: Resveratrol-induced apoptotic death in human U251 glioma cells. Mol Cancer Ther 4: 554-561, 2005.

11. Tseng SH, Lin SM, Chen JC, et al: Resveratrol suppresses the angiogenesis and tumor growth of gliomas in rats. Clin Cancer Res 10: 2190-2202, 2004.

12. Yang YP, Chang YL, Huang PI, et al: Resveratrol suppresses tumorigenicity and enhances radiosensitivity in primary glioblastoma tumor initiating cells by inhibiting the STAT3 axis. J Cell Physiol 227: 976-993, 2012.

13. Zhang W, Wang YE, Zhang Y, et al: Global epigenetic regulation of microRNAs in multiple myeloma. PLoS One 9: e110973, 2014.

14. Chan JA, Krichevsky AM and Kosik KS: MicroRNA-21 Is an Antiapoptotic Factor in Human Glioblastoma Cells. Cancer Res 65: 6029-6033, 2005.

15. Tuomarila M, Luostari K, Soini Y, et al: Overexpression of microRNA-200c predicts poor outcome in patients with PR-negative breast cancer. PLoS One 9: e109508, 2014.

16. Wang KY, Ma J, Zhang FX, et al: MicroRNA-378 inhibits cell growth and enhances 1-OHP-induced apoptosis in human colorectal cancer. IUBMB Life 66: 645-654, 2014.

17. Chen Q, Yang L, Xiao Y, et al: Circulating microRNA-182 in plasma and its potential diagnostic and prognostic value for pancreatic cancer. Med Oncol 31: 225, 2014.

18. Yiwei Li, Dejuan Kong, Zhiwei Wang, et al: Regulation of microRNAs by natural agents: an emerging field in chemoprevention and chemotherapy research. Pharm Res 27: 1027-1041, 2010.

19. Melkamu T, Zhang X, Tan J, et al: Alteration of microRNA expression in vinyl-carbamateinduced mouse lung tumors and modulation by the chemopreventive agent indole-3-carbinol. Carcinogenesis 31: 252-258, 2010.

20. Li Y, VandenBoom TG, Kong D, et al: Up-regulation of miR-200 and let-7 by natural agents leads to the reversal of epithelialto-mesenchymal transition in gemcitabine-resistant pancreatic cancer cells. Cancer Res 69: 6704-6712, 2009.

21. Sun M, Estrov Z, Ji Y, et al: Curcumin (diferuloylmethane) alters the expression profiles of microRNAs in human pancreatic cancer cells. Mol Cancer Ther 7: 464-473, 2008.

22. Jia Z, Wang K, Wang G, et al: MiR-30a-5p antisense oligonucleotide suppresses glioma cell growth by targeting SEPT7. PLoS One 8: e55008, 2013.

23. Jia Z, Wang K, Zhang A, et al: MiR-19a and miR-19b overexpression in gliomas. Pathol Oncol Res 19: 847-853, 2013.

24. Zhou X, Ren Y, Moore L, et al: Downregulation of miR-21 inhibits EGFR pathway and suppresses the growth of human glioblastoma cells independent of PTEN status. Lab Invest 90: 144-155, 2010.

25. Pu P, Liu X, Liu A, et al: Inhibitory effect of antisense epidermal growth factor receptor RNA on the proliferation of rat C6 glioma cells in vitro and in vivo. J Neurosurg 92: 132-139, 2000.

26. Pu P, Kang C, Li J, et al: The effects of antisense AKT2 RNA on the inhibition of malignant glioma cell growth in vitro and in vivo. J Neurooncol 76: 1-11, 2006.

27. Bove K, Lincoln DW and Tsan MF: Effect of resveratrol on growth of 4T1 breast cancer cells in vitro and in vivo. Biochem Biophys Res Commun 291: 1001-1005, 2002.

28. Kuwajerwala N, Cifuentes E, Gautam S, et al: Resveratrol induces prostate cancer cell entry into $S$ phase and inhibits DNA synthesis. Cancer Res 62: 2488-2492, 2002.

29. Kim YA, Lim SY, Rhee SH, et al: Resveratrol inhibits inducible nitric oxide synthase and cyclooxygenase-2 expression in betaamyloid-treated C6 glioma cells. Int J Mol Med 17: 1069-1075, 2006. 
30. Tili E, Michaille JJ, Alder H, et al: Resveratrol modulates the levels of microRNAs targeting genes encoding tumor-suppressors and effectors of TGF $\beta$ signaling pathway in SW480 cells. Biochem Pharmacol 80: 2057-2065, 2010.

31. Sheth S, Jajoo S, Kaur T, et al: Resveratrol reduces prostate cancer growth and metastasis by inhibiting the Akt/MicroRNA-21 pathway. PLoS One 7: e51655, 2012.

32. Krichevsky AM and Gabriely G: miR-21: a small multi-faceted RNA. J Cell Mol Med 13: 39-53, 2009.

33. Papagiannakopoulos T, Shapiro A and Kosik KS: MicroRNA-21 targets a network of key tumor-suppressive pathways in glioblastoma cells. Cancer Res 68: 8164-8172, 2008.

34. Zhu S, Wu H, Wu F, et al: MicroRNA-21 targets tumor suppressor genes in invasion and metastasis. Cell Res 18: 350-359, 2008.

35. Wang F, Li T, Zhang B, et al: MicroRNA-19a/b regulates multidrug resistance in human gastric cancer cells by targeting PTEN. Biochem Biophys Res Commun 434: 688-694, 2013.

36. Zawrocki A and Biernat W: Epidermal growth factor receptor in glioblastoma. Folia Neuropathol 43: 123-132, 2005.

37. Pu P, Kang C, Zhang Z, et al: Downregulation of PIK3CB by siRNA suppresses malignant glioma cell growth in vitro and in vivo. Technol Cancer Res Treat 5: 271-280, 2006.

38. Gürsel DB, Connell-Albert YS, Tuskan RG, et al: Control of proliferation in astrocytoma cells by the receptor tyrosine kinase/PI3K/ AKT signaling axis and the use of PI-103 and TCN as potential anti-astrocytoma therapies. Neuro Oncol 13: 610-621, 2011.

39. Ruano Y, Mollejo M, Camacho FI, et al: Identification of survivalrelated genes of the phosphatidylinositol 3'-kinase signaling pathway in glioblastoma multiforme. Cancer 112: 1575-1584, 2008.

40. Kang CS, Pu PY, Li YH, et al: An in vitro study on the suppressive effect of glioma cell growth induced by plasmid-based small interference RNA (siRNA) targeting human epidermal growth factor receptor. J Neurooncol 74: 267-273, 2005.

41. Brantley EC and Benveniste EN: Signal transducer and activator of transcription-3: a molecular hub for signaling pathways in gliomas. Mol Cancer Res 6: 675-684, 2008.

42. Germain D and Frank DA: Targeting the cytoplasmic and nuclear functions of signal transducers and activators of transcription 3 for cancer therapy. Clin Cancer Res 13: 5665-5669, 2007.

43. Quoc Trung L, Espinoza JL, Takami A, et al: Resveratrol induces cell cycle arrest and apoptosis in malignant NK cells via JAK2/ STAT3 pathway inhibition. PLoS One 8: e55183, 2013.
44. Han L, Yue X, Zhou X, et al: MicroRNA-21 expression is regulated by $\beta$-catenin/STAT3 pathway and promotes glioma cell invasion by direct targeting RECK. CNS Neurosci Ther 18: 573-583, 2012.

45. Sminia P, Stoter TR, van der Valk P, et al: Expression of cyclooxygenase-2 and epidermal growth factor receptor in primary and recurrent glioblastoma multiforme. J Cancer Res Clin Oncol 131: 653-661, 2005.

46. Banerjee S, Bueso-Ramos C and Aggarwal BB: Suppression of 7,12-dimethylbenz(a)anthracene-induced mammary carcinogenesis in rats by resveratrol: role of nuclear factor-kappaB, cyclooxygenase-2, and matrix metalloprotease 9. Cancer Res 62: 4945-4954, 2002.

47. Ji Q, Liu X, Fu X, et al: Resveratrol inhibits invasion and metastasis of colorectal cancer cells via MALAT1 mediated Wnt//3-catenin signal pathway. PLoS One 8: e78700, 2013.

48. Pu P, Zhang Z, Kang C, et al: Downregulation of Wnt 2 and betacatenin by siRNA suppresses malignant glioma cell growth. Cancer Gene Ther 16: 351-361, 2009.

49. Filippi-Chiela EC, Thomé MP, Bueno e Silva MM, et al: Resveratrol abrogates the temozolomide-induced G2 arrest leading to mitotic catastrophe and reinforces the temozolomideinduced senescence in glioma cells. BMC Cancer 22: 147, 2013.

50. Nakada M, Furuta T, Hayashi Y, et al: The strategy for enhancing temozolomide against malignant glioma. Front Oncol 2: 98 , 2012.

51. Huang H, Lin $\mathrm{H}$, Zhang $\mathrm{X}$, et al: Resveratrol reverses temozolomide resistance by downregulation of MGMT in T98G

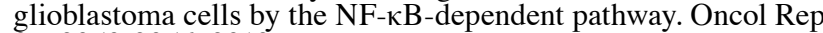
27: 2050-2056, 2012

52. Yuan Y, Xue X, Guo RB, et al: Resveratrol enhances the antitumor effects of temozolomide in glioblastoma via ROS-dependent AMPK-TSC-mTOR signaling pathway. CNS Neurosci Ther 18: 536-546, 2012.

53. Gagliano N, Aldini G, Colombo G, et al: The potential of resveratrol against human gliomas. Anticancer Drugs 21: 140-150, 2010.

54. Iguchi K, Toyama T, Ito T, et al: Antiandrogenic activity of resveratrol analogs in prostate cancer LNCaP cells. J Androl 33: 1208-1215, 2012.

55. Szekeres T, Saiko P and Fritzer-Szekeres M: Chemopreventive effects of resveratrol and resveratrol derivatives. Ann NY Acad Sci 1215: 89-95, 2011. 